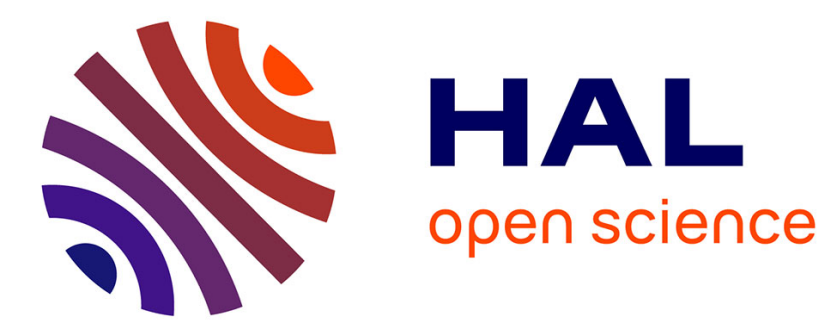

\title{
Thermal fluctuations of the contact line
}

\author{
Y. Pomeau
}

\section{To cite this version:}

Y. Pomeau. Thermal fluctuations of the contact line. Journal de Physique Lettres, 1983, 44 (14), pp.585-592. 10.1051/jphyslet:019830044014058500 . jpa-00232235

\section{HAL Id: jpa-00232235 https://hal.science/jpa-00232235}

Submitted on 1 Jan 1983

HAL is a multi-disciplinary open access archive for the deposit and dissemination of scientific research documents, whether they are published or not. The documents may come from teaching and research institutions in France or abroad, or from public or private research centers.
L'archive ouverte pluridisciplinaire HAL, est destinée au dépôt et à la diffusion de documents scientifiques de niveau recherche, publiés ou non, émanant des établissements d'enseignement et de recherche français ou étrangers, des laboratoires publics ou privés. 
Classification

Physics Abstracts

05.40

\title{
Thermal fluctuations of the contact line
}

\author{
Y. Pomeau \\ Service de Physique Théorique, CEN-Saclay, 91191 Gif sur Yvette Cedex, France
}

(Reçu le 3 mars 1983, révisé le 20 mai, accepté le 25 mai 1983)

\begin{abstract}
Résumé. - On étudie les fluctuations thermiques de la ligne de contact dans un champ de pesanteur, et l'on montre qu'elles divergent dans l'approximation quadratique si la phase liquide mouille le solide. Toutefois cette divergence ne doit pas être considérée comme une divergence critique au sens habituel.

Abstract. - The thermal fluctuations of the contact line in a gravity field are studied, and it is shown that they diverge in the quadratic approximation if the liquid phase wets the solid. Nevertheless this divergence cannot be really considered as a critical divergence in the usual sense.
\end{abstract}

In this note we discuss the effect of thermal fluctuations on a contact line. We shall consider the case of a semi infinite liquid vapour [1] interface meeting a vertical solid plane.

When a fluid-fluid interface joins a solid surface, the contact angle between the two surfaces is determined by the Young-Dupré equation [2]

$$
\gamma_{\mathrm{vl}} \cos \theta=\gamma_{\mathrm{vs}}-\gamma_{\mathrm{ls}}
$$

where $\gamma_{a b}(a, b=1, v$ or $s$-liquid, vapour or solid) is the energy per unit area of the $a / b$ interface at equilibrium. We shall consider the thermal fluctuations around this macroscopic picture of the three-phase boundary. Our strategy will be the following : we shall first compute the energy change due to the interaction between the fluid and the solid and show that the Young-Dupre condition follows from the extremalization of this energy with respect to variations in the position of the contact line. The second variation of this energy shall allow us to define the EinsteinSmoluchowski statistical weight for the thermal fluctuations of the contact line. It will appear in particular that, if the liquid wets the solid, this second variation of the energy vanishes so that - in a certain sense - the fluctuations of the contact line are "critical ", although this idea must be considered carefully.

In the spirit of the Einstein-Smoluchowski approach, we shall consider fluctuations with a long range only for which continuum mechanics still applies. In this framework, parameters such as surface tension or mass density keep the same definition and value as for macroscopic phenomena. We shall not try to relate them to melocular interactions (a hard task anyway). In this description, the wetting transition [6] corresponds to a situation for which the Antonov relation between the capillary constants is satisfied. The interest of the present approach could be also in the explicit account for gravity and in the fact that long range fluctuations might be 
easier to observe with light beams (see our point (3) at the end) than phenomena at a molecular level.

To begin with, and fix our notations, let us consider the mechanical problem (i.e. without thermal fluctuations) of the merging of a liquid/vapour $(1 / v)$ interface with a vertical solid. Let $x$ be the (horizontal) coordinate perpendicular to the solid and $h(x)$ be the height of the $1 / v$ interface. We take $h(+\infty)=0$ as the " sea level ", determined by the plane $1 / v$ interface far from the solid. The total energy coming from the deformation of this plane interface by the interaction between the fluid and the solid is $E\{h\}$. It is the sum of three contributions :

$$
E\{h\}=E^{(1)}+E^{(2)}+E^{(3)} \text {. }
$$

The energy $E^{(1)} \equiv \gamma_{1 \mathrm{v}} \int_{0}^{\infty} \mathrm{d} x\left(\left(1+h_{x}^{2}\right)^{1 / 2}-1\right)$ is due to the deformation of the $1 / \mathrm{v}$ interface with respect to its plane configuration, $x=0$ being the Cartesian equation of the solid surface and $h_{x} \equiv \mathrm{d} h / \mathrm{d} x$. The energy $E^{(2)} \equiv \rho g \int_{0}^{\infty} \mathrm{d} x \frac{h^{2}(x)}{2}$ is due to the ascension of the fluid into the gravity field. We have denoted as $g$ the gravity acceleration, and as $\rho$ the difference between the mass densities of the liquid and of the vapour. The energy $E^{(3)}$ comes from the wetting of the solid surface by the fluid. If $h_{0}[\equiv h(x=0)]$ is the height of the contact line above the « sea level $"$, the surface energy due to the wetting is $E^{(3)}=\left(\gamma_{1 \mathrm{~s}}-\gamma_{\mathrm{vs}}\right) h_{0}$ : it is obtained by subtracting from the actual value of the surface energy due to the fluid-solid interaction a standard (and $h_{0}$-independent) value, that is the value of this energy if $h_{0}=0$. In the preceding expression of the energy, we omitted everywhere the integration over the horizontal coordinate $(y)$ parallel to the solid surface. This is because we assumed that $h$ is $y$-independent, and had only to consider energies per unit length along $y$.

The form of the interface, i.e. the $x$-dependence of $h(x)$, is determined by the Euler-Lagrange equation $\frac{\delta}{\delta h(x)}\left(E^{(1)}+E^{(2)}\right)=0$. It reads

$$
h_{x x}=\left(\frac{\rho g}{\gamma_{1 v}}\right) h\left(1+h_{x}^{2}\right)^{3 / 2}
$$

or, by taking the capillary length $\left(\gamma_{1 v} / \rho g\right)^{1 / 2}$ as unit :

$$
h_{x x}=h\left(1+h_{x}^{2}\right)^{3 / 2} \text {. }
$$

With the boundary condition $h=0$ and $h_{x}=0$ at $x=+\infty$, this yields after the first integration :

$$
1-\frac{h^{2}}{2}=\left(1+h_{x}^{2}\right)^{-1 / 2}
$$

As said before, the height of the contact line, i.e. $h_{0}$, is determined by the condition that the energy $E$ is stationary under the variations of $h_{0}, h(x)$ itself being a solution of the autonomous equation 3.

A little algebra shows that

$$
E^{(1)}+E^{(2)}=\int_{0}^{h_{0}} \cdot \frac{\mathrm{d} h}{\left|h_{x}\right|}\left(\left(1+h_{x}^{2}\right)^{1 / 2}-1+\frac{h^{2}}{2}\right)
$$

where the unit energy had been taken as $\gamma_{1 v}$ times the capillary length times a standard length in the $y$-direction. 
As $h_{x}$ is given by equation 3 , one deduces from (4) that

$$
E^{(1)}+E^{(2)}=\int_{0}^{h_{0}} \mathrm{~d} h h\left(1-\frac{h^{2}}{4}\right)^{1 / 2}=\frac{4}{3}\left(1-\left(1-\frac{h_{0}^{2}}{4}\right)^{3 / 2}\right)
$$

at equilibrium.

The condition of stationarity of the total energy under changes in the height of the contact line is

and it gives

$$
\frac{\mathrm{d}}{\mathrm{d} h_{0}}\left(E^{(1)}+E^{(2)}+E^{(3)}\right)=0,
$$

with

$$
G=h_{0}\left(1-\frac{h_{0}^{2}}{4}\right)^{1 / 2}
$$

$$
G \equiv \frac{\gamma_{1 \mathrm{~s}}-\gamma_{\mathrm{vs}}}{\gamma_{\mathrm{lv}}}
$$

To check that this is equivalent to the Young-Dupré condition $G=\cos \theta$, one needs the relations

and

$$
\left.h_{x}\right|_{x=0}=-\operatorname{cotg} \theta
$$

$$
1-\frac{h_{0}^{2}}{2}=\left.\left(1+h_{x}^{2}\right)^{-1 / 2}\right|_{x=0}=\sin \theta \text {. }
$$

The so-called perfect wetting is realized if $\theta=0$ and corresponds to $G=1$ and $h_{0}=\sqrt{2}$.

To calculate the thermal fluctuations of $h_{0}$ near its equilibrium mean value $\tilde{h}_{0}$, one must expand $E$ near $\widetilde{h}_{0}$ up to the second order in $\delta \equiv h_{0}-\widetilde{h}_{0}$, where $h_{0}$ is fixed by the Young-Dupré condition. Let us assume for the moment that $\delta$ does not depend on $y$. The calculation is straightforward and one deduces from (5) that

$$
E\left(\tilde{h}_{0}+\delta\right)-E\left(\tilde{h}_{0}\right) \underset{\delta \rightarrow 0}{\simeq} \frac{\delta^{2}}{2} \frac{\left(1-\frac{\tilde{h}_{0}^{2}}{2}\right)}{\left(1-\frac{\tilde{h}_{0}^{2}}{4}\right)^{1 / 2}}-\frac{\delta^{3}}{6} \frac{\tilde{h}_{0}}{\left(1-\frac{\tilde{h}_{0}^{2}}{4}\right)^{3 / 2}}\left(\frac{3}{4}-\frac{\tilde{h}_{0}^{2}}{8}\right)+O\left(\delta^{4}\right)+\cdots
$$

Thus when the liquid wets the solid $\left(h_{0}^{2}=2\right)$, the thermal fluctuations of the contact line increase because the second variation of $E\left(h_{0}\right)$ at $\tilde{h}_{0}$ vanishes in this case. However, the situation is quite unusual here, compared to the usual case of critical fluctuations in bulk phases. If $\tilde{h}_{0}=\sqrt{2}$, the fluctuations with $\delta>0$ cannot be considered as fluctuations in the position of the contact line. If one imposes on the height $h_{0}$ that it must be larger than $\sqrt{2}$, the $1 / v$ interface minimizing the energy under this boundary condition is made, for $h \leqslant \sqrt{2}$, of the solution of (3) with $h_{x}=-\infty$ for $x=0$. And, for $\sqrt{2}<h \leqslant \sqrt{2}+\delta(>0)$ the $1 / v$ interface sticks to the vertical solid, so that the physical ( $\simeq$ observable) contact line is still at the height $\sqrt{2}$ above the "sea level".

Indeed this equation 7 does describe the variations of $E$ if $h_{0}$ is $y$-independent. Before considering the effects of this possible $y$-dependence we shall deal in a hypothetical 2-dimensional world, where this effect should be absent. The Einstein-Smoluchowski weight for the equilibrium distribution of $h_{0}$ should be there $\sim \exp -\frac{1}{k_{\mathrm{B}} T}\left(E^{(1)}+E^{(2)}+E^{(3)}\right), k_{\mathrm{B}}$ being the Boltzmann 
constant and $T$ the absolute temperature. Under these conditions, the mean height of the contact line is

$$
\tilde{h}_{0}=\left.k_{\mathrm{B}} T \frac{\partial}{\partial G} \operatorname{Ln} Z\right|_{T}
$$

with

$$
Z \equiv \int_{0}^{\sqrt{2}} \mathrm{~d} h \exp -\frac{1}{k_{\mathrm{B}} T}\left(\frac{4}{3}\left(1-\left(1-\frac{h^{2}}{4}\right)^{3 / 2}\right)-G h\right) .
$$

The equation 6 is obtained from these expressions in the low temperature limit $(T \rightarrow 0)$ by looking at the stationary phase in this integral. If $|G|<1$, this stationary phase is attained at the height defined by equation 6 . If the liquid wets the solid, one must take $G=1$. Actually, the condition $G>1$ has no meaning at equilibrium : a thin liquid film covers the solid surface and the "apparent $\gamma_{\mathrm{sv}}$ becomes $\gamma_{\mathrm{sl}}+\gamma_{\mathrm{lv}}$, the stationary phase is at the upper boundary $(\sqrt{2})$ of the integral in (8) and one finds in the low temperature expansion :

$$
\tilde{h}_{0} \simeq \sqrt{2}-\left(3 k_{\mathrm{B}} T\right)^{1 / 3} \frac{\Gamma(2 / 3)}{\Gamma(1 / 3)}+\cdots
$$

where $\Gamma($.$) is the usual transcendent. In the same limit (T \rightarrow 0)$, the order of magnitude of the thermal fluctuation of $h_{0}$ is $\left(k_{\mathrm{B}} T\right)^{1 / 3}$, as the first correcting term on the right hand side of (9). As shown below, the order of magnitude of these fluctuations is $\left(k_{\mathrm{B}} T\right)^{1 / 2}$ outside the wetting conditions.

Let us consider now the real (3d) world, where $h_{0}$ may depend, a priori, on the horizontal coordinate $y$ parallel to the solid wall. To make the calculations possible, we shall assume that the typical length of variation of $h_{0}$ along $y$ is much larger than 1 (= physically much larger than the capillary length). The computation of the lowest order energy variation due to this $y$-dependence of $h_{0}$ is greatly simplified by noting that any change in the total energy proportional to first order variations of $h$ vanishes, due to the Euler-Lagrange equations. The lowest order for $h(x, y)$ is the " adiabatic " solution $H\left(h_{0}(y), x\right)$ where $H\left(h_{0}, x\right)$ is the solution of (3) with the boundary condition $h(x=0)=h_{0}$. The equation for $h(x, y)$ extending the equation 2 to situations with a $y$-dependence is

$$
h_{x x}+h_{y y}+h_{x x} h_{y}^{2}+h_{y y} h_{x}^{2}-2 h_{x} h_{y} h_{x y}=h\left(1+h_{x}^{2}+h_{y}^{2}\right)^{3 / 2} .
$$

The smallness parameter in this expansion is formally the derivative with respect to $y(\partial / \partial y)$, and no term linear in $\partial / \partial y$ appears in equation 10.

Thus, these linear terms are absent too in the expansion of $h(x, y)$. And from all these arguments the only source for the terms of order $h_{0, y}^{2}$ in the energy expansion is in the $h_{y}^{2}$ dependence of the $1 / v$ surface energy.

The adiabatic energy is in lowest order (in $\left.\partial_{y}\right)$

$$
E_{\mathrm{ad}}\left\{h_{0}\right\}=\int \mathrm{d} y\left(\frac{4}{3}\left(1-\left(1-\frac{h_{0}^{2}}{4}\right)^{3 / 2}\right)-G h_{0}\right)
$$

and the first correction due to a non-constant $h_{0}(y)$ is

$$
E\left\{h_{0}(y)\right\}-E_{\mathrm{ad}}\left\{h_{0}(y)\right\} \underset{\partial_{y} \rightarrow 0}{\simeq} \frac{1}{2} \int \mathrm{d} y \int \mathrm{d} x h_{y}^{2}\left(1+h_{x}^{2}\right)^{-1 / 2} .
$$

To compute the right hand side of (11) at the dominant order (in the smallness parameter $\partial_{y}$ ), one must insert into it the adiabatic solution. 
Thus, in this approximation

$$
h_{y} \simeq h_{0, y} H_{h_{0}}\left[h_{0}, x\right] .
$$

As equation 3 is autonomous with respect to $x$, we have

$$
H_{h_{0}}\left(h_{0}, x\right)=H_{x}\left(h_{0}, x\right) \cdot\left(h_{0, x}^{-1}\right),
$$

where $h_{0, x}$ is the derivative $h_{x}$ computed from (3) for $h=h_{0}$. Accounting now for all these remarks, one finds :

$$
\begin{aligned}
E\left\{h_{0}(y)\right\}-E_{\mathrm{ad}}\left\{h_{0}(y)\right\} \underset{\partial y \rightarrow 0}{\simeq} \frac{1}{2} \int \mathrm{d} y \frac{h_{0, y}^{2}}{h_{0, x}^{2}} \int_{0}^{h_{0}} \mathrm{~d} h\left|h_{x}\right|\left(1+h_{x}^{2}\right)^{-1 / 2}= \\
=\frac{4}{3} \int \mathrm{d} y h_{0, y}^{2} \frac{\left(1-\frac{h_{0}^{2}}{2}\right)^{2}}{h_{0}^{2}\left(1-\frac{h_{0}^{2}}{4}\right)} \cdot\left(1-\left(1-\frac{h_{0}^{2}}{4}\right)^{3 / 2}\right) .
\end{aligned}
$$

Thus, near $h_{0}=\sqrt{2}$ the term proportional to $h_{0, y}^{2}$ in the expression of the energy becomes very small. In particular it is much smaller than the quadratic term in the right hand side of (7), which is of order $\left(1-\frac{h_{0}^{2}}{2}\right)$, although the coefficient of $h_{0, y}^{2}$ is of order $\left(1-\frac{h_{0}^{2}}{2}\right)^{2}$. Thus to estimate the order of magnitude of the thermal fluctuations of $h_{0}$ in the wetting conditions, it appears reasonable to assume that the coherence length of the fluctuation along $y$ is the capillary length. The corresponding estimate for the dimensionalized order of magnitude of the thermal fluctuations of the height is

$$
\delta h \sim \frac{\left(k_{\mathrm{B}} T\right)^{1 / 3}}{\left(\gamma_{1 \mathrm{v}} \rho g\right)^{1 / 6}} .
$$

This is to be compared with their order of magnitude outside the wetting conditions, i.e. $\left(k_{\mathrm{B}} T / \gamma_{\mathrm{lv}}\right)^{1 / 2}$.

The following simple arguments allow us to find these estimates. Let $\delta h$ be the fluctuation of the contact line. In the wetting condition, near this contact line the $1 / v$ interface follows a parabolic cylinder of equation

$$
h(0)-h(x) \sim(x \lambda)^{1 / 2},
$$

where $\lambda$ is the capillary length $\left[=\left(\frac{\gamma_{1 \mathrm{v}}}{\rho g}\right)^{1 / 2}\right]$. Consider a fluctuation coherent over a length of order $\lambda$ along $y$, the corresponding change of gravitation energy is

$$
\rho g \chi^{2} \int_{h(\dot{0})-\delta h}^{h(0)} \mathrm{d} h x(h) \sim \rho g \lambda(\delta h)^{3},
$$

because $h(0) \sim \lambda$. The order of magnitude of the thermal fluctuations is now obtained by equating this with the energy «quantum » $k_{\mathrm{B}} T$. This yields :

$$
\delta h_{\mathrm{W}} \sim\left(\frac{k_{\mathrm{B}} T}{\rho g \lambda}\right)^{1 / 3} \sim \frac{\left(k_{\mathrm{B}} T\right)^{1 / 3}}{\left(\lambda g \delta_{1 \mathrm{v}}\right)^{1 / 6}} .
$$


In non-wetting condition this estimate changes, because of the replacement of $h(0)-h(x) \sim(x \lambda)^{1 / 2}$ by $h(0)-h(x) \sim x$ and one obtains

$$
\delta h_{\mathrm{N} . \mathrm{w} .} \sim\left(\frac{k_{\mathrm{B}} T}{\gamma_{\mathrm{lv}}}\right)^{1 / 2} .
$$

By eliminating $k_{\mathrm{B}} T$, one finds

$$
\delta h_{\mathrm{w}}^{3} \sim \lambda \delta h_{\mathrm{N} . \mathrm{w} .}^{2} \cdot
$$

As a conclusion let us raise a few points :

(1) The typical time constant in the dynamics of these fluctuations will be the diffusion time for the vorticity in the vicinity of the solid fluid interface, that is (capillary length) ${ }^{2} /($ kinematic viscosity).

(2) To make the thermal fluctuations larger, one may think of reducing $\gamma_{1 \mathrm{v} 2} \rho$ and/or $g$. In particular one might expect large fluctuations in microgravity experiments [4]. This could (perhaps) explain some puzzling differences [4] observed between experiments carried out in microgravity conditions and on Earth.

(3) Pierre Bergé [5] suggested a very clever way to observe these fluctuations, by using light propagating near the solid wall under the condition of partial reflection at the solid/vapour interface and total reflection at the solid/liquid interface. Thus the edge of the transmitted beam through the fluid should fluctuate, due to the contact line fluctuations. The suggestion leads to the difficult question of the structure of this diffraction edge .

(4) To show that the thermal fluctuations of the contact line, as we considered them, are " independent " of other fluctuations of the $1 / v$ interface, consider an arbitrary fluctuation $\delta h(x)$ of the interface (to simplify the matter, we limit ourselves to a $2 \mathrm{~d}$ geometry), so that the contact point fluctuation is $\delta h(0)$. Let us split $\delta h(x)$ as

so that

$$
\delta h(x)=\delta_{1} h(x)+\delta_{2} h(x),
$$

$$
\delta_{1} h(x) \equiv \delta h(0) h_{x}\left[\left.h_{x}\right|_{x=0}\right]^{-1}
$$

is the part of $\delta h(x)$ due to the change of position of the contact point. We want to show first that, in the quadratic approximation, no cross term as $\delta_{1} h(x) \delta_{2} h(x)$ appears in the energy. The second variation of the energy $E\{h\}$ is

$$
\delta^{2} E=\frac{1}{2} \int_{0}^{\infty} \mathrm{d} x\left(\delta h^{2}(x)+\left(1+h_{x}^{2}\right)^{-3 / 2} \delta h_{x}^{2}\right),
$$

the sought cross term being

$$
\int_{0}^{\infty} \mathrm{d} x\left(\delta_{1} h \delta_{2} h+\left(1+h_{x}^{2}\right)^{-3 / 2} \delta_{1} h_{x} \delta_{2} h_{x}\right)
$$

or

$$
\int_{0}^{\infty} \mathrm{d} x \delta_{2} h\left[\delta_{1} h-\left(\left(1+h_{x}^{2}\right)^{-3 / 2} \delta_{1} h_{x}\right)_{x}\right] .
$$

To obtain this last form one integrates by part with the condition $\delta_{2} h(0)=0$. As $\delta_{1} h(x) \sim h_{x}$, $\delta_{1} h(x)=\left(\left(1+h_{x}^{2}\right)^{-3 / 2} \delta_{1} h_{x}\right)_{x}$ follows from equation 2 by derivation and the cross term vanishes.

This shows that $\delta_{1} h$ and $\delta_{2} h$ are independent fluctuations in this order. If one wants to account for cubic terms in the energy, to describe accurately the contact line fluctuations in the wetting 
conditions, terms such as $\delta_{1} h^{2}(x) \delta_{2} h(x)$ or $\delta_{1} h(x) \delta_{2} h^{2}(x)$ have to be included in the energy expansion. They could modify the quantitative picture of the large fluctuations of the contact line in the wetting conditions, but not their divergence in the quadratic approximation. Notice also that the fluctuations $\delta_{2} h$ could also be important for determining the details of the diffraction edge alluded to in the previous point (3).

(5) Another more subtle point should affect the picture of the contact line fluctuations in the wetting conditions. Under these conditions, the fluctuations of the $1 / v$ interface smoothly join those of the liquid film physisorbed on the solid substrate above the contact line. Let $e(z)$ be its thickness. The corresponding energy is (in dimensionless units and for a $2 \mathrm{~d}$ geometry)

$$
E^{(\mathrm{f})}\{e\}=\int_{h_{0}}^{\infty} \mathrm{d} z\left(z e(z)+\left(1+e_{z}^{2}\right)^{1 / 2}\right) .
$$

Indeed the "mean-field theory " $(\sim T=0)$ predicts that $e(z)=0$ for $z>h_{0}$. As the thermal fluctuations have a meaning if $e(z)>0$ only, these " one sided " fluctuations around the stationary phase change the mean value of $e(z)$. Owing to this limitation to the range $e(z)>0$, a precise estimate of $\langle e(z)\rangle$ with the statistical weight $\exp \left(-\frac{E^{(\mathfrak{f})}\{e\}}{k_{\mathrm{B}} T}\right)$ is difficult. Using the same sort of argument as presented before for computing $\delta h$, we may guess that $\langle e(z)\rangle \sim 1 / z$ at $z \gg \lambda$. To show this, let us assume again that the coherence length of the fluctuations is $\lambda$ in the $z$-and $y$-direction. This is a natural hypothesis because there is no parameter left with the dimension of a length in $E^{(f)}(e)$, once the unit length has been chosen as $\lambda$. However, the thermal fluctuations could introduce a new length in the problem, besides the one which appears explicitly in the statistical weight. We shall assume that this is not the case. Under these conditions, a fluctuation of the film thickness at height $z(\gg \lambda)$ induces a change in gravitational energy of order $\rho . g . \lambda^{2} . z . e\left[\lambda^{2}=\lambda\right.$ (in the vertical direction, $\times \lambda($ along $y)$ )]. Equating this with $k_{\mathrm{B}} T$, one gets

$$
\langle e(z)\rangle \sim \frac{k_{\mathrm{B}} T}{\rho g \lambda^{2} z} \sim \frac{k_{\mathrm{B}} T}{\gamma_{1 \mathrm{v}} z} .
$$

This last result, although consistent within our approach, is in sharp disagreement with the basic assumption of our calculations, that is with the description of fluctuations by continuum mechanics. Actually the interfacial energy is such that

$$
\gamma_{\mathrm{lv}} \xi^{2} \sim k_{\mathrm{B}} T
$$

where $\xi$ is a microscopic correlation length. Whence the preceding result would predict

$$
z\langle e(z)\rangle \sim \xi^{2},
$$

and indeed $\langle e(z)\rangle\langle\xi$ if $z \gg \lambda \gg \xi$.

This shows the need of a microscopic theory in order to describe the transition of a wetting film to the horizontal $1 / v$ interface. Nevertheless, as the film thickness is certainly not much larger than $\xi$ this film cannot be seen as a piece of bulk phase, but merely as a specific $2 \mathrm{~d}$ phase.

The divergence of $\langle e(z)\rangle \sim 1 / z$ at $z=0$ is also in agreement with the fact that this approximation of a physisorbed film of microscopic thickness is no longer valid when this film joins the meniscus of macroscopic thickness. The precise way of merging of the two regions poses a non-trivial problem.

\section{Acknowledgments.}

I have benefited from discussions with and helpful criticisms from Pierre Bergé and Stanislas Leibler for this work. 


\section{References}

[1] Indeed the theory developed will be also valid for any interface between isotropic fluids (vapour or liquid) meeting a solid boundary provided the correct values of the capillary constants are taken into account.

[2] This picture of a well defined contact angle is inadequate for many experimental situations, due to hysteresis effects [3] attributed to heterogeneities of the solid surface at a molecular level. Nevertheless our theory applies at least to the case where one of the fluids wets the solid surface. In this last case, hysteresis phenomena seem to be absent [3].

[3] See, for instance Neumann, A. W., in Wetting, Spreading and Adhesion, J. F. Padday, ed. (Academic Press, London) 1978 ; and ref. [4].

[4] Malmejac, Y., Bewersdorff, A., De Riva, I. and Napolitano, L. G., Challenges and Prospectives of microgravity research in space, ESA report BR-05, October' 1981.

[5] Bergé, P., private communication.

[6] Brézin, E., Halperin, B. I. and Leibler, S., Phys. Rev. Lett. 50 (1983) 1387 and references quoted therein. 